Application of the CE/SE Method to a Two-Phase Detonation Model in Porous Media

This article has been downloaded from IOPscience. Please scroll down to see the full text article.

2011 Chinese Phys. Lett. 28030203

(http://iopscience.iop.org/0256-307X/28/3/030203)

View the table of contents for this issue, or go to the journal homepage for more

Download details:

IP Address: 159.226.231.78

The article was downloaded on 22/03/2012 at 06:30

Please note that terms and conditions apply. 


\title{
Application of the CE/SE Method to a Two-Phase Detonation Model in Porous Media*
}

\author{
DONG He-Fei(董贺飞) $)^{1}$, HONG Tao(洪滔) ${ }^{1 * *}$, ZHANG De-Liang(张德良) ${ }^{2}$ \\ ${ }^{1}$ Institute of Applied Physics and Computational Mathematics, Beijing 100094 \\ ${ }^{2}$ LHD, Institute of Mechanics, Chinese Academy of Sciences, Beijing 100190
}

(Received 21 October 2010)

We extend the conservation-element and solution-element method to simulate a two-phase detonation model in porous media. The accuracy of the method is validated by calculating an inert compaction problem. The main characteristics of piston-driven detonation phenomena, including the compaction wave, the onset of combustion, and the transition to detonation, could be predicted successfully.

PACS: 02.60.Cb, 47.40.Rs, 82.33.Vx $\quad$ DOI: $10.1088 / 0256-307 \mathrm{X} / 28 / 3 / 030203$

Two-phase detonation in porous media is an important process. However, it is very complicated in experiment and numerical simulation since the mechanism is not completely clear. Dynamic compaction is a fundamental phenomenon of two-phase detonation in porous media. Steady compaction waves were observed by Sandusky and Liddiard. ${ }^{[1]}$ in a piston-driven porous HMX bed. To understand compaction and detonation in porous media better, several two-phase continuum models have been developed ${ }^{[2-4]}$ The main challenge in the numerical simulation of two-phase detonation models is the method of capturing the strong discontinuity in the field. The most commonly used numerical methods are based on either the MacCormack scheme or Riemann solution. The MacCormack scheme needs strong artificial viscosity to capture shocks and maintain stability, which may reduce the accuracy of the results. The method based on the Riemann solution is complicated in constructing Riemann solvers. To overcome these difficulties, in this Letter we apply the space-time conservation-element and solution-element $(\mathrm{CE} / \mathrm{SE})$ method to simulate a two-phase detonation model.

The CE/SE method originally proposed by Chang, ${ }^{[5]}$ provides a new way to solve the hyperbolic conservation equation. It has many features differing from the existing well-established schemes. The $\mathrm{CE} / \mathrm{SE}$ method satisfies the local and global flux conservation in space and time and needs simple treatments of boundary conditions. Because of its simplicity and accuracy, the CE/SE method has been successfully applied to many fluid problems. Wu et al. ${ }^{[6]}$ applied the CE/SE method to the gas-phase chemical reaction flow. Wang et al. ${ }^{[7]}$ simulated the critical wedge angle of cellular detonation reflections. Zhang et $a l_{.}{ }^{[8]}$ studied the plume dynamics of a pulse detonation engine. Weng et al. ${ }^{[9,10]}$ solved a two-phase flow in interior ballistics and the problem of the pulse detonation engine. The simulation of chemical reac- tion flow by the $\mathrm{CE} / \mathrm{SE}$ scheme is mainly gas-phase. In this study, we extend the CE/SE scheme to the simulation of the solid energetic granular detonation problem.

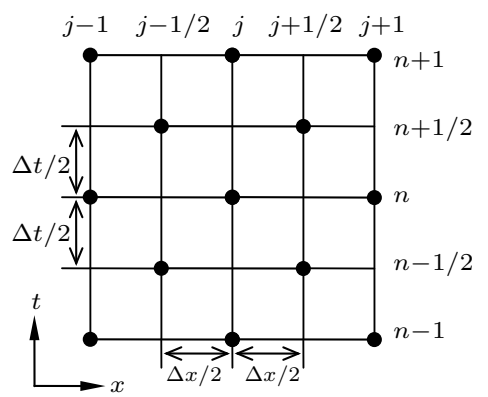

Fig. 1. Staggered space-time mesh of the CE/SE scheme.

The $\mathrm{CE} / \mathrm{SE}$ method treats time and space as one entity. The concepts of the SE and the CE are introduced. In the SE, variables are continuous. The conservation equation is integrated over the CE. Details are illustrated in Ref. [5]. Figure 1 shows the staggered space-time mesh of the computational region. For the conservation equation $\frac{\partial U}{\partial t}+\frac{\partial F(U)}{\partial x}=0$, the $\mathrm{CE} / \mathrm{SE}$ scheme reads

$$
\begin{aligned}
U_{j}^{n}= & \frac{1}{2}\left[U_{j-1 / 2}^{n-1 / 2}+\frac{\Delta x}{4}\left(U_{x}\right)_{j-1 / 2}^{n-1 / 2}+U_{j+1 / 2}^{n-1 / 2}\right. \\
& \left.-\frac{\Delta x}{4}\left(U_{x}\right)_{j+1 / 2}^{n-1 / 2}\right] \\
& +\frac{1}{2} \frac{\Delta t}{\Delta x}\left[F_{j-1 / 2}^{n-1 / 2}+\frac{\Delta t}{4}\left(F_{t}\right)_{j-1 / 2}^{n-1 / 2}\right. \\
& \left.-F_{j+1 / 2}^{n-1 / 2}-\frac{\Delta t}{4}\left(F_{t}\right)_{j+1 / 2}^{n-1 / 2}\right]
\end{aligned}
$$

where $U_{x}=\frac{\partial U}{\partial x}$ and $F_{t}=\frac{\partial F}{\partial t}$ denote spatial and temporal gradients of the variables.

The spatial gradients of the variables can be obtained simultaneously,

$$
\left(U_{x}\right)_{j}^{n}=\frac{\left|U_{x}^{+}\right|^{\alpha} U_{x}^{-}+\left|U_{x}^{-}\right|^{\alpha} U_{x}^{+}}{\left|U_{x}^{+}\right|^{\alpha}+\left|U_{x}^{-}\right|^{\alpha}},
$$

\footnotetext{
*Supported by the NSAF under Grant No 10676120.

**To whom correspondence should be addressed. Email: hongtao@iapcm.ac.cn

(C) 2011 Chinese Physical Society and IOP Publishing Ltd
} 
where

$$
U_{x}^{ \pm}= \pm \frac{U_{j \pm 1 / 2}^{n-1 / 2}-U_{j}^{n}+\Delta t\left(U_{t}\right)_{j \pm 1 / 2}^{n-1 / 2} / 2}{\Delta x / 2}
$$

with $\alpha=1-2$. The other gradients, such as $F_{x}, U_{t}$ and $F_{t}$, can be calculated by the chain rule and conservation equations.

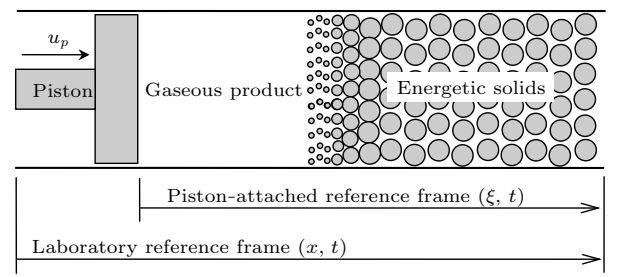

Fig. 2. Schematic of a piston-driven problem.

Figure 2 shows a simple schematic of a pistondriven problem in porous media. The piston moves with a constant velocity in the stationary granular bed. Adiabatic compression of the porous media provides energy to initiate the chemical reaction of solids. Solid mass, momentum, and energy are converted into gas mass, momentum, and energy. The two-phase model equations are given as follows:

$$
\begin{gathered}
\frac{\partial\left(\rho_{1} \phi_{1}\right)}{\partial t}+\frac{\partial\left(\rho_{1} \phi_{1} u_{1}\right)}{\partial x}=\Gamma \\
\frac{\partial\left(\rho_{1} \phi_{1} u_{1}\right)}{\partial t}+\frac{\partial\left(\rho_{1} \phi_{1} u_{1}^{2}+p_{1} \phi_{1}\right)}{\partial x}=\Gamma u_{2}-D, \\
\frac{\partial\left[\rho_{1} \phi_{1}\left(e_{1}+u_{1}^{2} / 2\right)\right]}{\partial t} \\
+\frac{\partial\left[\rho_{1} \phi_{1} u_{1}\left(e_{1}+u_{1}^{2} / 2+p_{1} / \rho_{1}\right)\right]}{\partial x} \\
=\Gamma\left(e_{2}+u_{2}^{2} / 2+q_{\mathrm{chem}}\right)-D u_{2}-Q \\
\frac{\partial\left(\rho_{2} \phi_{2}\right)}{\partial t}+\frac{\partial\left(\rho_{2} \phi_{2} u_{2}\right)}{\partial x}=-\Gamma \\
\frac{\partial\left(\rho_{2} \phi_{2} u_{2}\right)}{\partial t}+\frac{\partial\left(\rho_{2} \phi_{2} u_{2}^{2}+p_{2} \phi_{2}\right)}{\partial x}=-\Gamma u_{2}+D \\
\frac{\phi_{1} \phi_{2}}{\mu_{c}}\left[\left(p_{2}-p_{1}\right)-\left(p_{2 o}-p_{1 o}\right)\right. \\
\left.\frac{\phi_{2}^{2}}{\phi_{2 o}^{2}} \frac{\left(2-\phi_{2 o}\right)^{2}}{\left(2-\rho_{2} \phi_{2}\left(e_{2}+u_{2}^{2} / 2\right)\right]} \frac{\left.\partial \phi_{2}\right)}{\partial t}\right]-\frac{\Gamma}{\rho_{2}} \\
+\frac{\partial\left[\rho_{2} \phi_{2} u_{2}\left(e_{2}+u_{2}^{2} / 2+\phi_{2} / \rho_{2}\right)\right]}{\partial x} \\
=-\Gamma\left(e_{2}+u_{2}^{2} / 2\right)+D u_{2}+Q
\end{gathered}
$$

$$
\begin{gathered}
\frac{\partial n}{\partial t}+\frac{\partial\left(u_{2} n\right)}{\partial x}=0 \\
\frac{4}{3} \pi r^{3} n=\phi_{2}
\end{gathered}
$$

$$
\begin{aligned}
& \frac{\partial I}{\partial t}+u_{2} \frac{\partial I}{\partial x} \\
= & k_{I}(1-I) \exp \left[-\frac{T_{I}}{T_{1} \phi_{1}+T_{2} \phi_{2}}\right] \\
& \cdot\left[\frac{p_{1} \phi_{1}+p_{2} \phi_{2}-p_{1 o} \phi_{1 o}-p_{2 o} \phi_{2 o}}{p_{1 o} \phi_{1 o}+p_{2 o} \phi_{2 o}}\right]^{2} .
\end{aligned}
$$

In these equations, the subscripts 1 and 2 denote the variables of the gas and solid, respectively. The subscript $o$ represents the initial state. The variables are as follows: $\rho$ the density, $u$ the velocity; $p$ the pressure, $e$ the specific internal energy, $\phi\left(\phi_{1}+\phi_{2}=1\right)$ the volume fraction, $n$ the number of particles per unit volume, and $r$ the radius of the spherical solid particles. The variable $I(0<I<1)$ is an ignition parameter introduced by Gonthier and Powers ${ }^{[11]}$ to model the induction period occurring prior to the onset of combustion in piston-driven detonation experiments. In the source terms of the equations, $\Gamma=\frac{3}{r} \rho_{2} \phi_{2} a p_{1}^{m} H\left(I-I_{i g}\right)$ is the interphase mass transfer, $H$ is the Heaviside function, $D=\beta \frac{\phi_{1} \phi_{2}}{r}\left(u_{1}-u_{2}\right)$ is the interphase drag force, $Q=h \frac{\phi_{1} \phi_{2}}{r^{1 / 3}}\left(T_{1}-T_{2}\right)$ is the interphase convection heat transfer, $q_{\text {chem }}$ is granular chemical energy, $T$ is the temperature, $I_{i g}, \mathrm{k}_{I}$ and $\mathrm{T}_{I}$ are the constant parameters associated with ignition. The equations of state for the simulation are $e_{1}=c_{v 1} T_{1}, p_{1}=\rho_{1} R_{1} T_{1}\left(1+b \rho_{1}\right), e_{2}=c_{v 2} T_{2}+\frac{\rho_{20} \sigma}{\gamma_{2} \rho_{2}}$, $p_{2}=\left(\gamma_{2}-1\right) c_{v 2} \rho_{2} T_{2}-\frac{\rho_{20} \sigma}{\gamma_{2}}$.

To deal with the moving boundary of the piston, Eqs. (3)-(12) in the laboratory reference frame $(x, t)$ are transformed into a piston-attached reference frame $(\xi, t)$. The transformation is given by $\xi=x-\int u_{p} d t$ and $v_{i}=u_{i}-u_{p}(i=1,2)$, where $u_{p}$ is the piston velocity. In this study, we assume the piston moved in a constant velocity of $100 \mathrm{~m} / \mathrm{s}$.

To verify the accuracy of our method, we first simulate an inert compaction problem. We presume that the chemical reaction would not occur in the granular solids. The interactions of gas and solid are only coupled with the inter-phase drag and inter-phase heat transfer. The computational domain is $0<\xi<0.5 \mathrm{~m}$ with 501 meshes. The initial conditions and the model parameters used for the simulation are given as $\rho_{1 o}=10 \mathrm{~kg} / \mathrm{m}^{3}, \rho_{2 o}=1900 \mathrm{~kg} / \mathrm{m}^{3}, T_{o}=300 \mathrm{~K}$, $\phi_{2 o}=0.73, r_{o}=1.0 \times 10^{-4} \mathrm{~m}, c_{v 1}=2400 \mathrm{~J} /(\mathrm{kg} \cdot \mathrm{K})$, $R_{1}=850 \mathrm{~J} /(\mathrm{kg} \cdot \mathrm{K}), b=1.1 \times 10^{-3} \mathrm{~m}^{3} / \mathrm{kg}, c_{v 2}=$ $1500 \mathrm{~J} /(\mathrm{kg} \cdot \mathrm{K}), \gamma_{2}=5, \sigma=8.98 \times 10^{6} \mathrm{~m}^{2} / \mathrm{s}^{2}, \beta=$ $1 \times 10^{4} \mathrm{~kg} /\left(\mathrm{m}^{2} \cdot \mathrm{s}\right), h=1 \times 10^{7} \mathrm{~J} /\left(\mathrm{K} \cdot \mathrm{s} \cdot \mathrm{m}^{8 / 3}\right), \mu_{c}=$ $1000 \mathrm{~kg} /(\mathrm{m} \cdot \mathrm{s}), q_{\text {chem }}=0$.

Figure 3 shows the numerical results of the velocity (relative to the laboratory reference frame), volume fraction and pressure of the granular solids. Here $\xi$ is 
the position to the piston. A compaction wave quickly developed and propagated away with a steady speed of $411 \mathrm{~m} / \mathrm{s}$. The solid volume fraction and pressure for the steady wave are 0.964 and $64.5 \mathrm{MPa}$. These values of the compaction wave speed $D$, the solid volume fraction $\phi_{2}$ and the pressure $p_{2}$ agree well with the pre- vious reports, ${ }^{[1,11,12]}$ which are given in Table 1 . We simulate the case on a finer grid of 1000 meshes for further validation. The calculated compaction wave trace is shown in Fig. 4. The result is independent of the grid number.
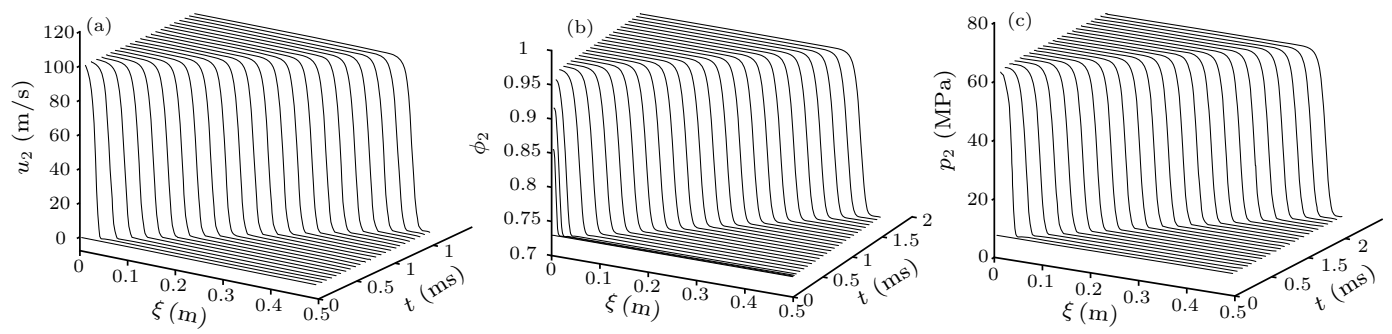

Fig. 3. Numerical results of the compaction wave in granular solids: (a) velocity, (b) volume fraction, and (c) pressure.

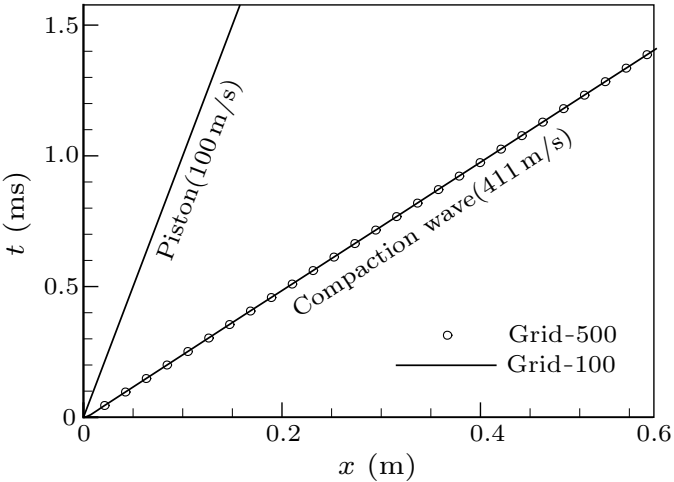

Fig. 4. Calculated compaction trace.

We numerically simulate the detonation process based on the inert compaction problem. Here the granular solids begin to combust when the ignition variable $I$ exceeds a certain threshold. Equation (12) is an evolution equation for the ignition variable. It models the ignition variable as an increasing function of pressure and temperature of the gas and solid. ${ }^{[11]}$ The computational domain is $0<\xi<0.5 \mathrm{~m}$ with 501 meshes. The initial conditions and the model parameters used for the simulation are given as $\rho_{1 o}=10 \mathrm{~kg} / \mathrm{m}^{3}, \rho_{2 o}=1710 \mathrm{~kg} / \mathrm{m}^{3}, T_{o}=300 \mathrm{~K}$, $\phi_{2 o}=0.7, r_{o}=1.0 \times 10^{-4} \mathrm{~m}, c_{v 1}=2400 \mathrm{~J} /(\mathrm{kg} \cdot \mathrm{K})$, $R_{1}=850 \mathrm{~J} /(\mathrm{kg} \cdot \mathrm{K}), b=1.1 \times 10^{-3} \mathrm{~m}^{3} / \mathrm{kg}, c_{v 2}=$ $1500 \mathrm{~J} /(\mathrm{kg} \cdot \mathrm{K}), \gamma_{2}=5, \sigma=8.98 \times 10^{6} \mathrm{~m}^{2} / \mathrm{s}^{2}$, $\beta=1 \times 10^{4} \mathrm{~kg} /\left(\mathrm{m}^{2} \cdot \mathrm{s}\right), h=1 \times 10^{7} \mathrm{~J} /\left(\mathrm{K} \cdot \mathrm{s} \cdot \mathrm{m}^{8 / 3}\right)$, $\mu_{c}=100 \mathrm{~kg} /(\mathrm{m} \cdot \mathrm{s}), q_{\mathrm{chem}}=5.84 \times 10^{6}, a=2.9 \times$ $10^{-9} \mathrm{~m} /(\mathrm{Pa} \cdot \mathrm{s}), m=1, I_{i g}=0.5, k_{I}=1.0 \times 10^{6} \mathrm{~s}^{-1}$, and $T_{I}=2690 \mathrm{~K}$.

Table 1. Comparison of the present results with the data in references.

\begin{tabular}{ccccc}
\hline Values & Sandusky $^{[1]}$ & Gonthier $^{[11]}$ & Yang $^{[12]}$ & Present \\
\hline$D(\mathrm{~m} / \mathrm{s})$ & 432 & 418 & 402 & 411 \\
$\phi_{2}$ & 0.952 & 0.96 & 0.958 & 0.964 \\
$P_{2}(\mathrm{MPa})$ & 62.1 & 67.1 & 66.3 & 64.5 \\
\hline
\end{tabular}

Figure 5 shows the velocity (relative to the laboratory reference frame), density and pressure of the gaseous product. A compaction wave quickly forms due to the moving piston. The velocity and density increase to $100 \mathrm{~m} / \mathrm{s}$ and $67 \mathrm{~kg} / \mathrm{m}^{3}$, respectively. The pressure increases to $20 \mathrm{MPa}$ from the initial value of 2.57 MPa. As time goes on, the ignition is predicted to occur at the piston surface. The velocity, temperature and pressure increase rapidly. The detonation forms subsequently and strengthens in the propagation process. Lastly, the accelerating detonation grows into a steady detonation with a speed of $6160 \mathrm{~m} / \mathrm{s}$.
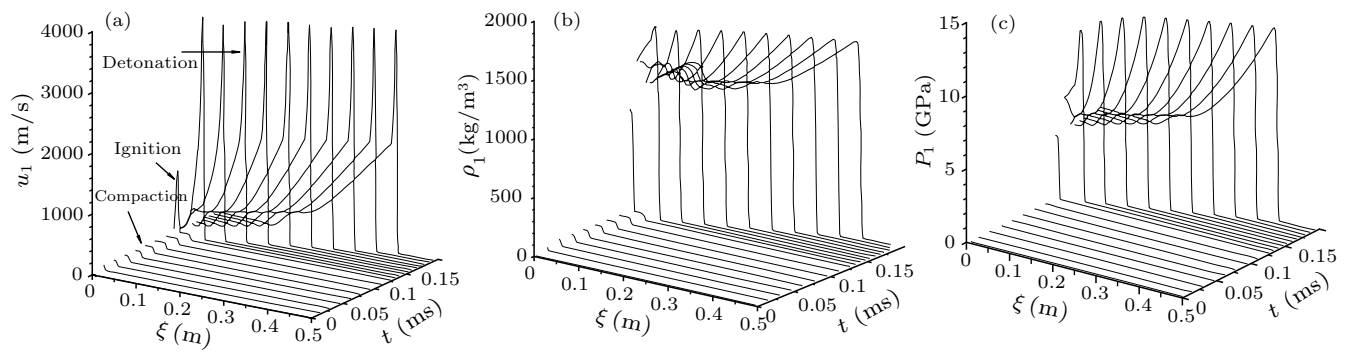

Fig. 5. Numerical results of the gaseous product: (a) velocity, (b) density, and (c) pressure. 
Figure 6 shows the gas velocity profile at $t=0.2 \mathrm{~ms}$. The velocity increases rapidly in the reaction zone due to the detonation. The detonation is a rarefaction wave which reduces the velocity at the end of the reaction zone $\left(\phi_{2}=0\right)$ to that of the piston. In front of the piston, a compaction zone is produced and the gas velocity is equal to the piston velocity. An interaction zone is predicted between the compaction zone and the rarefaction zone.

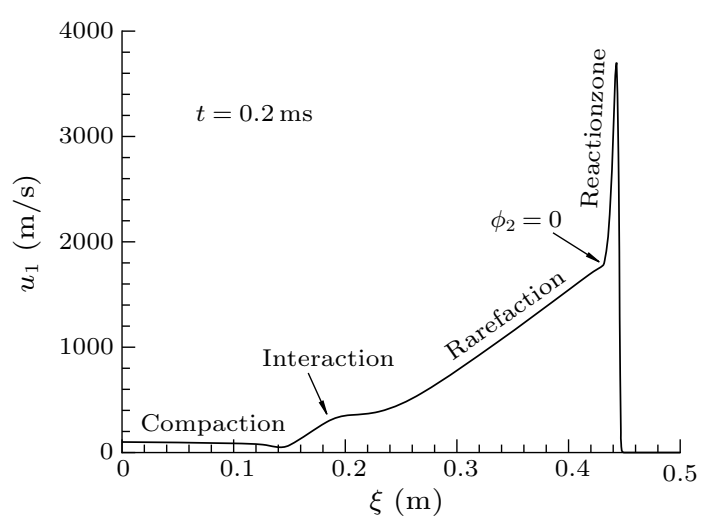

Fig. 6. The gas velocity profile at $t=0.2 \mathrm{~ms}$.

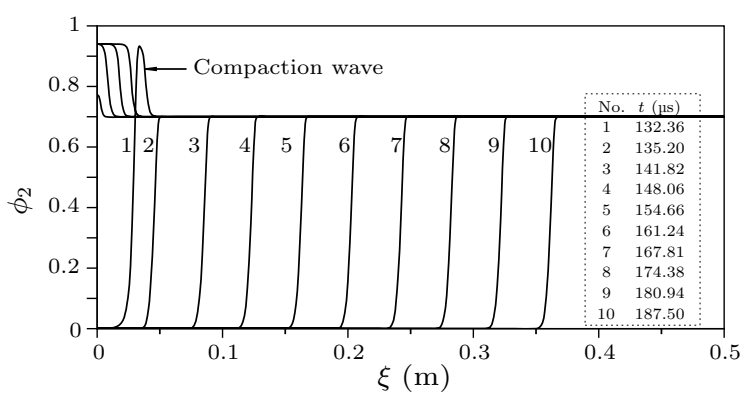

Fig. 7. Evolution of the solid volume fraction.

Figure 7 shows the evolution of the solid volume fraction. At the beginning of the piston movement, the granular solids are compacted from a volume fraction of 0.7 to 0.94 . The inert compaction wave propagates away from the piston with a speed of $295 \mathrm{~m} / \mathrm{s}$. After about $128 \mu \mathrm{s}$, the combustion is predicted to occur. The solid volume fraction continuously decreases. The granular near the piston surface is completely consumed at about $132 \mu \mathrm{s}$. After about $135 \mu \mathrm{s}$ the detonation overtakes the compaction wave and propagates steadily.

Table 2 is the comparison of the numerical results with the experiment and numerical simulation, which are cited from Ref. [11] directly. The values of the compaction wave speed $D_{c}$ (relative to the laboratory reference frame), the solid volume fraction behind the lead compaction wave $\phi_{2}$ and the detonation wave speed $D_{d}$ agree very well with each other.

Table 2. Comparison of the results with Ref. [11].

\begin{tabular}{cccc}
\hline Values & Experiment $^{[11]}$ & Simulation $^{[1]}$ & Present \\
\hline$D_{c}(\mathrm{~m} / \mathrm{s})$ & 400 & 401.98 & 395 \\
$\phi_{2}$ & 0.90 & 0.94 & 0.94 \\
$D_{d}(\mathrm{~m} / \mathrm{s})$ & 6200 & 6169.4 & 6160 \\
\hline
\end{tabular}

In conclusion, the $\mathrm{CE} / \mathrm{SE}$ method is applied to simulate a two-phase detonation model in porous media. The accuracy of the method is validated by comparing with other researches. An inert compaction problem is calculated first, the development and the structure of the compaction wave are validated by the data of the published experiment and simulations. A two-phase detonation process is numerically simulated by introducing a chemical reaction parameter. The numerical results have indicated the main characteristic of the detonation phenomenon, including the compaction wave, the rarefaction wave, the onset of combustion, and the transition to detonation, induced by low-velocity impact.

\section{References}

[1] Sandusky H W and Liddiard T P 1985 NSWC Technical Report 83-246 (NAVAL Surface Weapons Center, White Oak, MD 26 December 1985)

[2] Baer M R and Nunziato J W 1986 Int. J. multiphase Flow 12861

[3] Powers J M, Stewart D S and Krier H 1990 Combust. Flame 80264

[4] Stewart D S, Asay B W and Prasad K 1994 Phys. Fluids 62515

[5] Chang S C 1995 J. Comput. Phys. 119295

[6] Wu Y, Ma F and Yang V 2004 Int. J. Comput. Fluid Dynamics 18277

[7] Wang G, Zhang D L and Liu K X 2010 Chin. Phys. Lett. 27024701

[8] Zhang Z C, Yu S T, He H and Jorgenson P C E 2001 AIAA 2001-3614, 37th AIAA/ASME/SAE/ASEE JPC Conference (Salt Lake City, Utah 8-11 July 2001)

[9] Bai Q D and Weng C S 2008 Mech. Engin. 3021 (in Chinese)

[10] Wang J and Weng C S 2009 J. Gun Launch and Control 0353 (in Chinese)

[11] Gonthier K A and Powers J M 2000 J. Comput. Phys. 163 376

[12] Yang T 1995 J. Propulsion Technol. 1668 (in Chinese) 